\title{
Regional Lung Function Following Upper Sleeve Lobectomy for Bronchogenic Carcinoma
}

\author{
Hiroyoshi Ayabe, Yutaka Tagawa, Hiroharu Tsuji, \\ Tadayuki Oka, Shinsuke Hara, Katsunobu Kawahara \\ and Masao Tomita \\ The First Department of Surgery, Nagasaki University \\ School of Medicine, Nagasaki 852
}

Ayabe, H., Tagawa, Y., Tsuji, H., Oka, T., Hara, S., Kawahara, K. and Tоміта, M. Regional Lung Function Following Upper Sleeve Lobectomy for Bronchogenic Carcinoma. Tohoku J. Exp. Med., 1995, $176(1), 45-52-$ Regional lung function of the operated side following upper sleeve lobectomy ( $n=$ 8 ) and simple upper lobectomy $(n=7)$ for lung cancer was evaluated. Regional ventilation was studied with Krypton $81 \mathrm{~m}$ and regional pulmonary blood flow was studied with Technetium 99m. Measurements were taken from 12 to 24 months after operation. The ventilation rate of the operated side following right upper sleeve lobectomy $(n=5)$ was $42.9 \pm 6.7 \%$ and the perfusion rate was $37.4 \pm 4.6 \%$. The regional ventilation rate of the operated side after simple right upper lobectomy $(n=3)$ was $45.9 \pm 10.5 \%$ and the perfusion rate was $46.2 \pm 5.2 \%$. For the patients with left upper sleeve lobectomy $(n=3)$, these ratios were $29.9 \pm 11.1 \%$ and $19.2 \pm 3.6 \%$, respectively. For the patients with simple left upper lobectomy $(n=4)$, these ratios were $27.3 \pm 4.5 \%$ and $22.3 \pm 3.3 \%$. There were no stastical differences between the group with upper sleeve lobectomy and that with simple upper lobectomy. Regional function improved gradually with time. In conclusion, the regional lung function of the operated side following sleeve lobectomy was well preserved and showed no difference when compared with the regional function after simple lobectomy for lung cancer._— sleeve lobectomy; regional lung ventilation; regional lung perfusion; lung cancer

Lobectomy is considered a standard operative procedure for lung cancer, but in cases where cancer has invaded into the proximal bronchus, pneumonectomy is often performed. Although pneumonectomy has a high success rate as an operation for lung cancer, it carries a high risk of causing postoperative complications such as lung edema, broncho-pleural fistula and respiratory failure and has a high operative death rate (Nagasaki et al. 1982; Ginsberg et al. 1983). Accordingly, bronchoplasty came to be performed because it allows a high degree of preservation of pulmonary parenchyma and has a high success rate. Bronchoplasty has a lower operative mortality rate and a better prognosis than pneumonectomy and the results have been as good as those of lobectomy (Jensik et al. 1972; Bennet

Received September 12, 1994; revision accepted for publication February 23, 1995. 
and Smith 1978; Ayabe et al. 1982; First et al. 1987). However, not much has been reported on the degree to which lung function of the operated side is preserved after bronchoplasty.

Although there have been some reports on postoperative lung function by measuring overall pulmonary function using spirometry, not many studies have examined the lung function of operated side alone. This study reports on the results of the evaluation of the lung function of the operated side using pulmonary scintigraphy.

\section{Patients and Methods}

The subjects of this study were 8 patients who underwent upper sleeve lobectomy ( 5 patients on the right and 3 on the left) and 7 patients who underwent simple upper lobectomy (4 patients on the right and 3 on the left) for lung cancer. The operative procedure was decided on the basis of whether invasion of cancer existed at the proximal bronchus (upper and main bronchus). All operations were performed via a posterolateral thoracotomy. Mediastinal lymph node dissection was performed on all 15 cases. The two groups were matched for age, sex, location of the tumor and preoperative lung function (Table 1). At the time of post-operative pulmonary scanning measurements, local recurrence, distant metastasis and other pulmonary complications were not observed in any of the patients.

The pulmonary scanning measurements were performed from 12 months until

TABLE 1. Preoperative characteristic of patients

\begin{tabular}{lcc}
\hline & Sleeve & Simple \\
\hline Number of patients & 8 & 7 \\
Age (years) & $65 \pm 9.8$ & $56 \pm 5.8$ \\
Sex & & \\
Male & 7 & 6 \\
Female & 1 & 1 \\
Operative procedures & & \\
RUL & 5 & 3 \\
LUL & 3 & 4 \\
Spirometry & & \\
VC(L) & $3.51 \pm 0.46$ & $3.87 \pm 0.71$ \\
VC $(\%)$ & $109.9 \pm 6.1$ & $114.5 \pm 12.8$ \\
FEV & $2.04 \pm 0.55$ & $2.50 \pm 0.47$ \\
FEV & & $68.7 \pm 9.7$ \\
\hline
\end{tabular}

RUL, right upper lobectomy; LUL, left upper lobectomy; VC, Vital capacity; $\mathrm{FEV}_{1.0}$, forced expiratory volume in the first second; FVC, forced vital capacity. 
two years after the thoracotomy. In four patients, measurements were periodically taken for two years after the operation. The method of pulmonary ventilation scintigraphy involved patients inhaling Krypton $\left({ }^{81 \mathrm{~m}} \mathrm{Kr}\right.$ diluted in $0.1-0.2$ liter/min oxygen) through nasal canulae continuously in the sitting position. Under normal breathing, scanning was performed with an Ohio-Nuclear Sigma 410 (Ohio-Nuclear, Solon, OH, USA) and mean examination time was 5 min. For pulmonary circulation scintigraphy, technetium (Tc) perfusion rates were calculated by scanning with an Ohio-Nuclear Sigma 4104 to 5 min intravenous injection of ${ }^{99 m} \mathrm{Tc}$ macroaggregated albumin ( ${ }^{99 \mathrm{~m} T c M A A}$ ) $3 \mathrm{mCi}$ to patients in the supine position. Scanning was performed from both the front and back. The chest was divided into 4 sections from the upper part downward, and $\mathrm{Kr}$ and $\mathrm{Tc}$ uptake rates were calculated for each picture using computer transaction (Simis-4; Informatek, Paris, France).

The difference in distribution of the uptake of a given isotope between the operated side and the contralateral side was also examined using the mean values

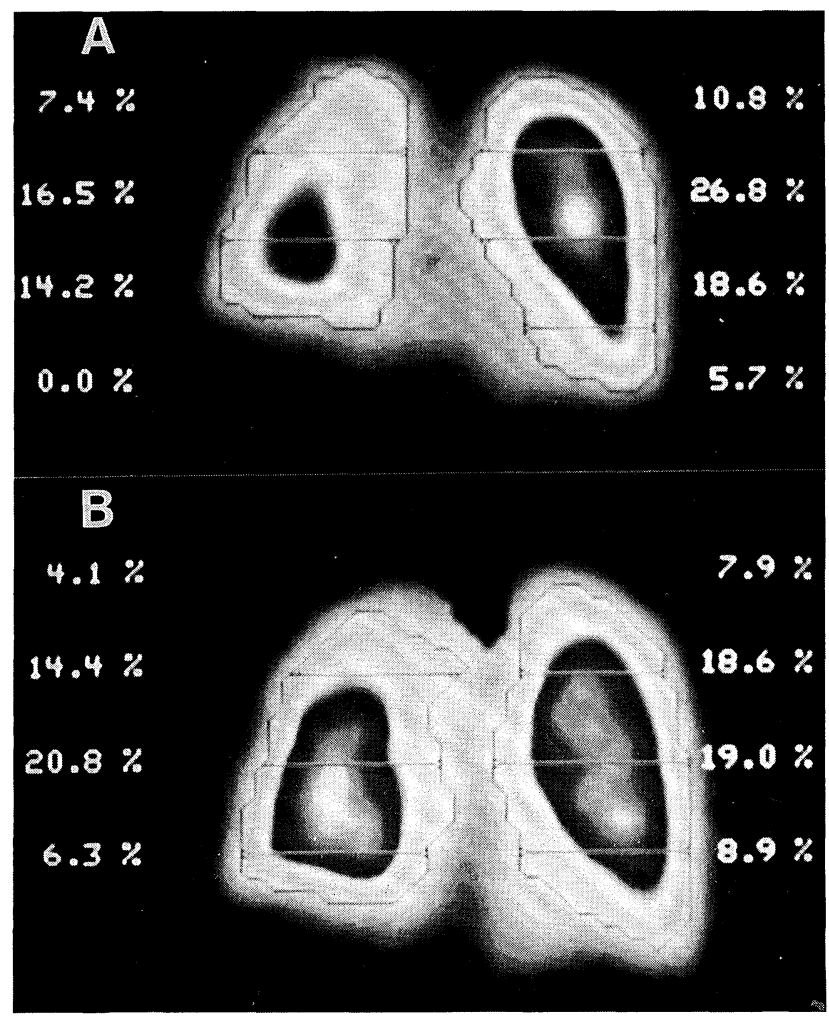

Fig. 1. Scanning of pulmonary ventilation (A) fnd perfusion (B) after right upper sleeve lobectomy. Scanning was performed from both the front and back. The chest was divided into 4 sections from the upper part downward and uptake rates were calculated for each picture. 
for front and back as the reference value (Fig. 1).

Using these measurements, pulmonary ventilation and pulmonary circulation of the patients with upper sleeve lobectomy and those with simple lobectomy were compared. Data are presented as mean \pm s.D. Statistical analysis was carried out by using the paired or unpaired $t$-test, as appropriate. For all tests, a probably value less than 0.05 was accepted as statistically significant.

\section{RESULTS}

In the patients with right upper sleeve lobectomy $(n=5)$, the mean Krypton ventilation rate of the operated side was $42.9 \pm 6.7 \%$. No significant difference was observed when compared to the patients with right simple upper lobectomy $(n=3)$ which had a mean Krypton ventilation rate of $45.9 \pm 10.5 \%$. The mean perfusion rate of the operated side following right upper sleeve lobectomy was $37.4 \pm 4.6 \%$ and that of the right simple upper lobectomy was $46.2 \pm 5.2 \%$. Although the perfusion rate was better in the latter group, the difference between the two groups was not significant. Comparing pulmonary ventilation and perfusion between the two operative procedures, in the patients with bronchoplasty a decline in perfusion was observed on the operated side (which was not the case with ventilation), but in those with simple lobectomy this decline was not observed (Fig. 2).

The mean ventilation rate of the operated side of the patients with left upper sleeve lobectomy $(n=3)$ was $29.9 \pm 11.1 \%$ and that of those with left simple upper lobectomy $(n=4)$ was $27.3 \pm 4.5 \%$, which showed no significant difference. The mean perfusion rate of the operated side after left upper sleeve lobectomy was $19.2 \pm 3.6 \%$ and that of those with left simple upper lobectomy was $22.3 \pm 3.3 \%$. Although the former group showed a lower rate, the difference was not significant. As was the case with right upper bronchoplasty, a decline in the perfusion of the operated side was observed in the left upper sleeve lobectomy (Fig. 3).

Pulmonary scanning measurements were periodically performed in four
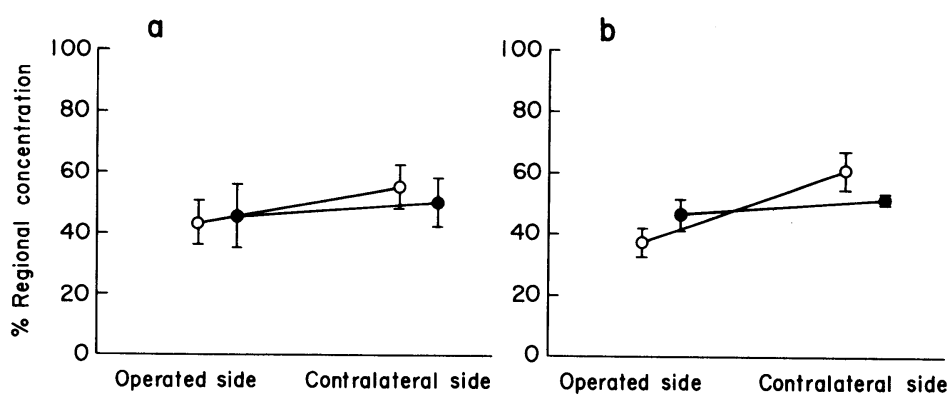

Fig. 2. Regional ventilation (a) and perfusion (b) of the operated side and contralateral side from 12 to 24 months after right upper sleeve $(\bigcirc-)$ ) and simple lobectomy $(\bullet \bullet)$. 

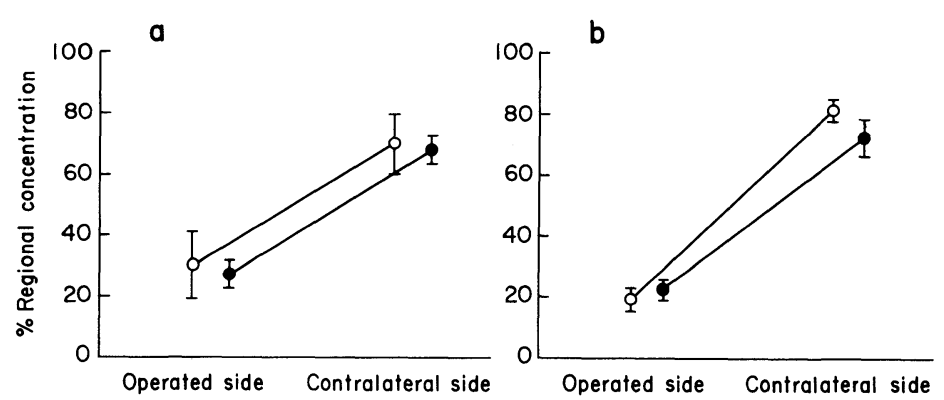

Fig. 3. Regional ventilation (a) and perfusion (b) of the operated side and contralateral side from 12 to 24 months after left upper sleeve $\left(\mathrm{O}_{-} \mathrm{O}\right)$ and simple lobectomy $(\bullet \bullet)$.

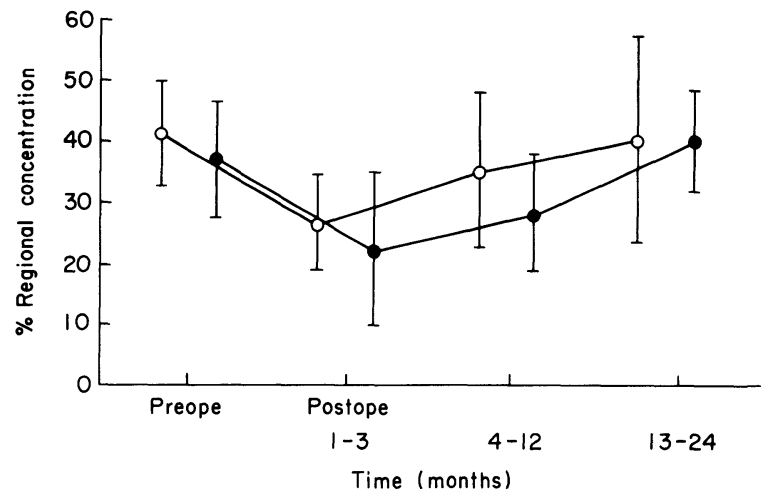

Fig. 4. Long-term changes of ventilation $\left(\mathrm{O}^{-} \mathrm{O}\right)$ and perfusion $(\bullet \bullet)$ rates of the operated side after upper sleeve lobectomy.

patients with bronchoplasty from one month to two years after the operation (Fig. 4). The mean ventilation rate during the period of one to 3 months after the operation was $26.5 \pm 7.8 \%$ and the mean perfusion rate was $20.7 \pm 12.6 \%$. In the 4 to 12 months postoperative period, the rates were $34.4 \pm 12.5 \%$ and $26.4 \pm 9.7 \%$, respectively, and during the 13 to 24 months post-operative period, they were $39.3 \pm 16.7 \%$ and $38.3 \pm 8.8 \%$. Improvement was observed in both rates, particularly in the long term perfusion rate after the operation.

\section{Discussion}

Sleeve lobectomy for lung cancer is performed when there is invasion into the left or right upper bronchus for the purpose of preserving pulmonary function. At first, it was mainly performed on compromised patients for whom pneumonectomy was not appropriate in the light of pulmonary function (Bennet and Smith 1978). However, it has been shown that the postoperative results of sleeve lobectomy were as good as those of simple lobectomy and better than those 
of pneumonectomy (Deslauriers et al. 1986). At present, sleeve lobectomy is performed on uncompromised patients.

Few studies have looked at pulmonary function following sleeve lobectomy. Studies have mainly measured total lung capacity (TLC), vital capacity, forced expiratory volume in the first second $\left(\mathrm{FEV}_{1.0}\right), \mathrm{FEV}_{1.0} \%$ and residual volume/ TLC and reported on total pulmonary function of the two lungs. Van Den Bosch et al. (1981) reported that vital capacity declined after the operation, but it was within the normal range in 25 of 32 cases $\left(78 \%\right.$ ) and no changes in $\mathrm{FEV}_{1.0} \%$ were observed between the pre- and post-operative values. Vanderhoeft (1978) also reported that although total lung capacity declined after the operation, it was still within the normal range and residual volume increased, while no differences between the pre- and post-operative values were observed in $\mathrm{FEV}_{1.0}$. Deslauriers et al. (1986) and Angeletti et al. (1991) also obtained similar results. Brusasco et al. (1988) compared the results of sleeve lobectomy and those of simple lobectomy using spirometry and reported that in the early postoperative period (two weeks after operation), $\mathrm{FEV}_{1.0}$ rate after surgery was $79 \%$ of the peroperative value in the former group and $63 \%$ in the latter group, which suggested that pulmonary function was sufficiently maintained after bronchoplastic lobectomies.

However, it is difficult to evaluate the pulmonary function of the operated side separately from the contralateral side using general spirometry. The pulmonary function of the operated side in the early postoperative period has been examined in experiments with dogs and reported by several researchers. Wood et al. (1974) reported that oxygen uptake of the operated lung significantly decreased in the early postoperative period after bronchoplastic operations and it took four weeks to recover. Sato (1977) reported that alveolar $\mathrm{O}_{2}$ of the operated lung significantly declined in the 14 to $21 \mathrm{hr}$ after the operation, but it gradually recovered to $80-90 \%$ of the preoperative value within 2 months. Minami (1980) reported that $A-a D_{02}$ of the operated lung significantly increased due to denervation in the perihilar area, which caused the decline of the oxygen uptake rate. It seemed that this functional deterioration is mainly due to denervation resulting from interruption of the plex in the bronchial wall and the pulmonary branch of the vagal nerve, as well as operative lung damage. The denervation may cause the decline of pulmonary blood flow, a dead-space-like effect due to retardation of blood flow and alveolar capillary block. It is considered that such deterioration, affected by denervation, gradually disappears by renervation in the peripheral bronchus. However, there are not many reports on the long-term post-operative pulmonary function of the operated side lung. In patients undergoing sleeve lobectomy, pulmonary scintigraphy using radioactive isotope is considered a useful method to evaluate the function of the reconstructed lung (Vanderhoeft 1978; Deslauriers et al. 1986; Ratto et al. 1986). Deslauriers et al. (1986) examined pulmonary ventilation with Xenon 133 scintigraphy and pulmonary perfusion with Technetium $99 \mathrm{~m}$ scintigraphy in 19 patients who had undergone 
sleeve lobectomy more than five years before. They reported that the mean pulmonary ventilation of the operated side was $48 \%$ and the mean pulmonary perfusion rate was $41 \%$ in the cases with right upper sleeve lobectomy while these rates were $40.8 \%$ and $29.3 \%$ in the cases with left upper sleeve lobectomy. In our study, the ventilatory capacity ratio of the operated side to the contralateral side was found to be $42.9 \%$ for right upper sleeve lobectomy and $29.3 \%$ for left upper sleeve lobectomy, and the perfusion rates were $37.4 \%$ for right and $18.3 \%$ for left upper sleeve lobectomy. Our results showed slightly lower rates than Deslauriers et al. (1986), and we believe that the difference in operative procedure (they did not perform mediastinal lymph node dissection) and the difference in postoperative period account for these slight difference. Ratto et al. (1986) compared the pulmonary function of the operated lung by pulmonary scintigraphy in cases with sleeve lobectomy and simple lobectomy and reported that there was no difference in Xenon 133 uptake rates of the operated side in the late post-operative stage between the two groups. These results suggest that the pulmonary function of the reconstructed lung was sufficiently maintained. It has also been shown that in the long-term, both ventilation and perfusion uptake rates of the reconstructed lung have increased gradually. This might be related to alleviation of postoperative pain of the chest wall, recovery of the reconstructed lung from surgical trauma and overinflation of the preserved lung due to decrease of the intrapleural residual space and pleural effusion (Brusasco et al. 1988; Angeletti et al. 1991).

Deslauriers et al. (1986) argued that the resection of poorly functioning lung, the prohibition of smoking and the more aggressive treatment for chronic lung disease after the operation were main reasons why the pulmonary function of the reconstructed lung following sleeve lobectomy was not severely altered. The decline in perfusion of the reconstructed lung observed in our study may be caused by the interruption of lymphatic vessels, parasympathetic nerves or bronchial circulation inherent in this operation (Wood et al. 1974) or an increased physiological dead space in the operated lung (Deslauriers et al. 1986; Brusasco et al. 1988). However, the mechanisms responsible for decreased pulmonary perfusion post sleeve lobectomy have not been identified and remain speculative to date.

In conclusion, the pulmonary function of the reconstructed lung is suffuciently maintained in the cases with sleeve lobectomy. Therefore, sleeve lobectomy should be used as an operative procedure for lung cancer when the preservation of pulmonary function and quality of life are taken into consideration.

\section{References}

1) Angeletti, C.A., Janni, A., Macchiarini, P., Ricagna, F. \& Pistelli, G. (1991) Functional results of bronchial sleeve lobectomy. Eur. J. Cardiothorac. Surg., 5, 410-413.

2) Ayabe, H., Nakamura, Y., Miura, T., Kugimiya, T., Koga, Y. \& Tsuji, Y. (1982) 
Bronchoplasty for bronchogenic carcinoma. World J. Surg., 6, 433-439.

3) Bennet, W.F. \& Smith, R.A. (1978) A twenty-year analysis of the results of sleeve resection for primary bronchogenic carcinoma. J. Thorac. Cardiovasc. Surg., 76, 840845.

4) Brusasco, V., Ratto, G.B., Crimi, P., Sacco, A. \& Motta, G. (1988) Lung function following sleeve lobectomy for bronchogenic carcinoma. Scan. J. Cardiovasc. Surg., 22, 73-78.

5) Deslauriers, J., Gaulin, P., Beaulieu, M., Piraux, M., Bernier, R. \& Cormier, Y. (1986) Long-term clinical and functional results of sleeve lobectomy for primary lung cancer. J. Thorac. Cardiovasc. Surg., 92, 871-879.

6) First, W.H., Mathisen, D.J., Hilgenberg, A.D. \& Grillo, H. (1987) Bronchial sleeve resection with and without pulmonary resection. J. Thorac. Cardiovasc. Surg., 93, 350-357.

7) Ginsberg, R.J., Hill, L.D., Eagan, R.T., Thomas, P., Mountain, C.F., Deslauriers, J., Fry, w.A., Butz, R.O., Goldberg, M., Waters, P.F., Jones, D.P. \& Pairolero, P. (1983) Modern thirty-day operative mortality for surgical resections in lung cancer. $J$. Thorac. Cardiovasc. Surg., 86, 654-658.

8) Jensik, R.J., Faber, L.P., Molloy, F.J. \& Amato, J.J. (1972) Sleeve lobectomy for carcinoma. A ten-year experience. J. Thorac. Cardiovasc. Surg., 64, 400-412.

9) Minami, H. (1980) An experimental study on post-operative functions of bronchial reconstructed lung-Especially the influence of vagal denervation-. Nagasaki Med. J., 55, 383-398.

10) Nagasaki, F., Flehinger, B.J. \& Martini, N. (1982) Complication of surgery in the treatment of carcinoma of the lung. Chest, 82, 25-29.

11) Ratto, G.B., Brusasco, V., Sacco, A., Villa, G., Mereu, C. \& Motta, G. (1986) Regional lung function following sleeve lobectomy. Pan. Med., 28, 335-338.

12) Sato, H. (1977) Study on the pathophysiological changes after bronchoplastic operation-with special reference to its effect on morphology as well as function of the reconstructed lung. J. Juzen Med. Soc., 86, 599-615.

13) Van Den Bosch, J.M.M., Bergstein, P.G.M., Laros, C.D., Gelissen, H.J., Riempst, S. \& Wagnaar, S.S. (1981) Lobectomy with sleeve resection in the treatment of tumors of the bronchus. Chest, 80, 154-157.

14) Vanderhoeft, P. (1978) Bronchoplastic lobectomies for advanced carcinoma: Functional changes and survival. Acta Chir. Belg., 4, 293-302.

15) Wood, P.B., Gilday, D., Illves, R., Rac, S. \& Pearson, F.G. (1974) A comparison of gas exchange after simple lobectomy and lobectomy with sleeve resection in dogs. $J$. Thorac. Cardiovasc. Surg., 68, 646-653. 